\title{
Novel mediators of breast cancer bone metastasis - insights from studies of gene-regulation and the global proteome
}

\author{
Janet E. Brown ${ }^{1}$, Steven L. Wood ${ }^{2}$ \\ ${ }^{1}$ Department of Oncology \& Metabolism, Weston Park Hospital, Sheffield, UK; ${ }^{2}$ Department of Oncology and Metabolism, The Medical School, \\ Sheffield, UK \\ Correspondence to: Professor Janet E. Brown. Department of Oncology \& Metabolism, Weston Park Hospital, Whitham Road, Sheffield, S10 2SJ, UK. \\ Email: j.e.brown@sheffield.ac.uk; Dr. Steven L. Wood. Department of Oncology and Metabolism, The Medical School, Beech Hill Road, Sheffield, \\ S10 2RX, UK. Email: s.l.wood@sheffield.ac.uk. \\ Comment on: Huang S, Chi Y, Qin Y, et al. CAPG enhances breast cancer metastasis by competing with PRMT5 to modulate STC-1 transcription. \\ Theranostics 2018;8:2549-64.
}

Submitted Oct 04, 2018. Accepted for publication Oct 16, 2018.

doi: $10.21037 /$ atm.2018.10.41

View this article at: http://dx.doi.org/10.21037/atm.2018.10.41

Breast cancer (BCa) is a leading cause of cancer death in women, being responsible for 458,000 deaths worldwide within women each year (1). Metastatic spread to the skeleton is a significant complication with over $75 \%$ of advanced breast cancer patients developing bone metastases (2). Current treatment options for breast cancer bone metastasis focus on the use of bone targeted agents such as denosumab (3) or bisphosphonates (4) to reduce the occurrence of skeletal related events, such as pathological fractures, hypercalcaemia or spinal cord compression. Adjuvant bisphosphonate use has demonstrated a significant reduction in risk of bone metastasis and improved survival within postmenopausal women (4). Despite these significant advances in the treatment of breast cancer bone metastasis there is still a significant unmet need for biomarkers which predict risk of breast cancer spread to bone, and the mechanisms responsible for spread to the skeleton are incompletely understood.

Cancer-spread to bone is known to alter bone homeostasis via signalling effects upon bone-resorbing osteoclasts and bone forming osteoblasts (5). Osteolytic metastases, which can occur within breast cancer, result from cancer cell secreted factors such as Parathyroid hormone related protein (PTHrP) triggering a "vicious cycle" of bone destruction. Osteoblastic metastases which occur in prostate and some breast cancer patients, involve osteoblast activation and phenomena such as endothelial-cell-to-osteoblast conversion, resulting in aberrant bone deposition (6).
Despite the pivotal importance of a supportive tumour stroma environment for development of bone metastatic cancers, molecular alterations within the tumour cells themselves are also important. Studies within models of human bone metastatic breast cancer established within immunocompromised mice, have identified sub-clones of breast cancer cells with enhanced ability to colonize bone and these have altered gene-expression signatures (7). In view of the importance of tumour-cell specific alterations in the establishment of bone metastases, high throughput techniques (including genomics, functional genomics and proteomics) have been applied to the identification of molecular alterations which promote cancer spread to bone. Such alterations have the potential to act as biomarkers predictive of bone metastasis risk. Quantitative proteomic comparison of parental and bone-homing MDA-MB-231 cells, identified a panel of proteins which were predictive of future bone metastasis risk, including the macrophage capping-protein (CapG) (8). This identification of CapG as a potential bone metastasis protein was subsequently validated within tissue microarrays from the large openlabel, international, multicentre, randomised, controlled, parallel-group phase 3 trial (AZURE) $(4,9)$. The AZURE trial compared women with stage II or III breast cancer randomly assigned to receive either standard adjuvant treatment or standard adjuvant treatment plus zoledronic acid with (at present) up to 10 years of follow-up, excellent clinical annotation and a significant proportion of cases 
developing bone metastases, making it an excellent resource for validation of bone-metastasis biomarkers. The level of CapG within this study was observed to be a predictor of bone metastasis risk within both an initial patient cohort and a subsequent validation set, in total comprising 689 patients all receiving standard treatment (8), and achieved even greater predictive ability when combined with a second biomarker PDZ-domain containing adaptor protein-GIPC1. Treatment with zoledronic acid was observed to eliminate the predictive effect of CapG levels suggesting that CapG-levels can also be used to inform patient treatment decisions (8). Despite these promising observations about the potential clinical relevance of CapG as a biomarker of bone metastasis risk the precise mechanism by which proteins such as CapG promote cancer cell spread to bone is currently unclear and the subject of active research.

In this edition Huang et al. have used state-of-the-art techniques for RNA and protein analysis to identify a key mechanism by which the protein macrophage-capping protein $(\mathrm{CapG})$ mediates breast cancer metastasis to bone. CapG was previously identified as a protein upregulated within highly metastatic variants of human breast cancer cells (10) and together with the PDZ-domain containing signalling adaptor protein GIPC1, levels of CapG and GIPC1 within primary breast tumours, have been observed to be predictive of breast cancer bone metastasis risk (8).

CapG has also been implicated in the spread to bone of other malignancies including lung and prostate cancer. Genome wide association studies within prostate cancer cell lines identified the CapG locus as the site of a key single nucleotide polymorphism regulating gene expression via chromatin modification (11). Silencing of CapG expression via siRNA has also been demonstrated to reduce the proliferation of prostate cancer cell-lines as well as regulating cellular apoptosis via the Caspase 6/9 pathway $(12,13)$. CapG has been identified as a major upregulated protein within lung adenocarcinomas with early recurrence compared to those without recurrence within 5 years of surgical resection, and immunohistochemical analysis of CapG expression within non-small cell lung cancer has been observed to be a significant prognostic indicator of disease severity and mortality $(14,15)$. Evidence was thus accumulated across several cancer types for a significant role of CapG within cancer cell proliferation and metastatic spread. In addition to a role within metastasis to bone, CapG expression is elevated within ocular melanomas as well as glioblastomas, cancers which are characterized by extensive local invasion and metastasis $(16,17)$.

Despite evidence that CapG plays a key role in cancer metastasis, the precise mechanism(s) by which the protein acts within bone metastasis are not clearly understood. CapG plays a key role in capping actin filaments within the cytoplasm and as such is a key regulator of diverse processes including cell-shape regulation and cell movement. Unlike other members of the gelsolin family of actin-assembly regulatory proteins, CapG lacks a nuclear export sequence and is thus localized both within the nucleus and the cytoplasm $(18,19)$. CapG shares homology with members of the basic helix-loop-helix family of transcription factors of which the c-Myc oncogene is a member (20). An early indication that CapG might have a nuclear role was the observation that introduction of a nuclear-export sequence into CapG reduced its ability to promote cancer cell invasion into collagen type-I and chicken heart fragments (19), furthermore point mutations within the nuclear export sequence which permitted nuclear re-entry were observed to restore the invasive ability of cells. Additional studies revealed that CapG entry into the nucleus was energy dependent and mediated via the cytosolic receptor importin- $\beta$. Despite this evidence the functions of CapG within the nucleus were otherwise poorly defined.

In the recent paper of Huang et al. (21) the mechanism by which CapG mediates increased bone metastasis via effects within the cell nucleus is significantly elucidated. Huang et al. demonstrate that CapG increases the transcription of the pro-metastatic gene stanniocalcin-I (STC-I). STC-I is a secreted glycoprotein hormone which has been previously demonstrated to be associated with poorsurvival within breast cancer, and in particular within basaltype breast cancer (22). Knockdown of STC-I expression has been demonstrated to inhibit both primary tumour formation and metastasis within murine models of human breast cancer (23). Huang et al. demonstrate that CapG mediates increased STC-1 expression by competing with the transcriptional repressor Arginine Methyltransferase-5 (PRMT5) for binding to the STC-I promoter. Decreased histone-4, Arginine-3 (H4R3) levels at the STC-I promoter following CapG-mediated displacement of PRMT5 result in increased transcription of STC-I. As would be expected from this mode of action, both CapG and PRMT5 levels are independent prognostic factors for breast cancer survival, with high CapG levels being associated with poorer patient outcomes, and high PRMT5 levels being associated with better patient prognosis (21).

This newly discovered function of CapG as a key epigenetic 
regulator further highlights the important role of epigenetic control within breast cancer bone metastasis. Previous studies have identified epigenetic control mechanisms playing a key role within bone metastasis including the regulation of hepatocyte-growth-factor (HGF)/Met signalling in breast cancer $(24,25)$. Epigenetic regulation confers on metastatic cancer cells the ability to respond to different external stimuli during the metastatic process. Epigenetic regulation of HGF/Met signalling results in both anoikis resistance and stem-cell characteristics characteristic of epithelial-mesenchymal transition (EMT) in metastatic breast cancer. CapG mediated upregulation of STC-I has the potential to modulate hypoxic responses within metastatic breast cancer cells $(26,27)$ as well as regulate expression of key pro-angiogenic regulators such as vascular endothelial growth factor (VEGF) $(28,29)$, functions integral to cancer spread to distant sites such as bone. STC-I is a key protein within calcium and phosphate homeostasis (30) as well as being a previously identified regulator of the generalised migratory ability of cancer cells, acting via the phosphoinositide-3-kinase (PI3K) pathway (31).

The epigenetic control mechanisms mediated by CapG further add to the body of evidence that epigenetic dysregulation is a feature of malignancy and a potential target for therapy. Histone deacetylases (HDACs) have entered advanced stage clinical trials and shown promising results within breast cancer when combined with ionizing radiation, cytotoxic agents or aromatase inhibitors (32-35). To date there have been fewer studies reported in which DNA methylation is used a therapeutic target in breast cancer. Despite this, alterations in DNA methylation have demonstrated utility as potential markers predictive of bone metastasis risk. Levels of promoter methylation are observed to be lower in breast cancer bone metastases than in brain metastases, and elevated levels of DNA-methyltransferase-I (DNMTI) are prognostic for shorter overall survival within bone metastasis (36). High-throughput profiling of global DNA-methylation patterns has identified panels of gene methylation which can be used to predict metastatic lethal progression within prostate cancer (37). The observation that CapG mediates STC-1 upregulation via altered methylation at the STC-I promoter thus adds an additional line of evidence for the utility of epigenetic markers within breast cancer.

The observation that CapG can act as both an actincapping protein with effects upon the cytoskeleton, and a transcriptional regulator with effects within the nucleus, emphasizes the important role of protein molecules and their unique ability to have pleiotropic effects within cells. Proteins originally associated with specific functions may act via additional, unexpected mechanisms to achieve the complex cellular changes required within metastasis. In addition, proteins implicated in organotropic metastasis may achieve their target organ specificity via downstream molecules. As an actin capping protein CapG does not appear to have an obvious link to bone-specific metastasis, but as an inducer of STC-I expression, a protein implicated in calcium and phosphate homeostasis, it's role within cancer spread to bone appears more rational. The example of CapG shows that our ability to identify biomarkers predictive of cancer spread to bone, also has the potential to reveal key cellular mechanisms by which cancer cells spread to, and colonize the bone micro-environment. These fundamental molecular insights into the development of bone metastasis will continue to open up new avenues for development of personalized medicine initiatives, as well as identify new targets for therapy within bone metastatic cancers.

\section{Acknowledgements}

None.

\section{Footnote}

Conflicts of Interest: The authors have no conflicts of interest to declare.

\section{References}

1. Torre LA, Bray F, Siegel RL, et al. Global cancer statistics, 2012. CA Cancer J Clin 2015;65:87-108.

2. Coleman RE. Metastatic bone disease: clinical features, pathophysiology and treatment strategies. Cancer Treat Rev 2001;27:165-76.

3. Lacey DL, Boyle WJ, Simonet WS, et al. Bench to bedside: elucidation of the OPG-RANK-RANKL pathway and the development of denosumab. Nat Rev Drug Discov 2012;11:401-19.

4. Wilson C, Bell R, Hinsley S, et al. Adjuvant zoledronic acid reduces fractures in breast cancer patients; an AZURE (BIG 01/04) study. Eur J Cancer 2018;94:70-8.

5. Guise TA. Breaking down bone: new insight into sitespecific mechanisms of breast cancer osteolysis mediated by metalloproteinases. Genes Dev 2009;23:2117-23. 
6. Lin SC, Yu-Lee LY, Lin SH. Osteoblastic Factors in Prostate Cancer Bone Metastasis. Curr Osteoporos Rep 2018. [Epub ahead of print].

7. Kang Y, Siegel PM, Shu W, et al. A multigenic program mediating breast cancer metastasis to bone. Cancer Cell 2003;3:537-49.

8. Westbrook JA, Cairns DA, Peng J, et al. CAPG and GIPC1: Breast Cancer Biomarkers for Bone Metastasis Development and Treatment. J Natl Cancer Inst 2016;108.

9. Coleman RE, Marshall H, Cameron D, et al. Breastcancer adjuvant therapy with zoledronic acid. N Engl J Med 2011;365:1396-405.

10. Xu SG, Yan PJ, Shao ZM. Differential proteomic analysis of a highly metastatic variant of human breast cancer cells using two-dimensional differential gel electrophoresis. J Cancer Res Clin Oncol 2010;136:1545-56.

11. Du M, Tillmans L, Gao J, et al. Chromatin interactions and candidate genes at ten prostate cancer risk loci. Sci Rep 2016;6:23202.

12. Li T, Hong X, Zhao J, et al. Gelsolin-like actin-capping protein is associated with patient prognosis, cellular apoptosis and proliferation in prostate cancer. Biomark Med 2016;10:1251-60.

13. Li BK, Guo K, Li CY, et al. Influence of suppression of CapG gene expression by siRNA on the growth and metastasis of human prostate cancer cells. Genet Mol Res 2015;14:15769-78.

14. Zhu WY, Hunag YY, Liu XG, et al. Prognostic evaluation of CapG, gelsolin, P-gp, GSTP1, and Topo-II proteins in non-small cell lung cancer. Anat Rec (Hoboken) 2012;295:208-14.

15. Ha ES, Choi S, In KH, et al. Identification of proteins expressed differently among surgically resected stage I lung adenocarcinomas. Clin Biochem 2013;46:369-77.

16. Lal A, Lash AE, Altschul SF, et al. A public database for gene expression in human cancers. Cancer Res 1999;59:5403-7.

17. Van Ginkel PR, Gee RL, Walker TM, et al. The identification and differential expression of calciumbinding proteins associated with ocular melanoma. Biochim Biophys Acta 1998;1448:290-7.

18. Watari A, Takaki K, Higashiyama S, et al. Suppression of tumorigenicity, but not anchorage independence, of human cancer cells by new candidate tumor suppressor gene CapG. Oncogene 2006;25:7373-80.

19. De Corte V, Van Impe K, Bruyneel E, et al. Increased importin-beta-dependent nuclear import of the actin modulating protein CapG promotes cell invasion. J Cell
Sci 2004;117:5283-92.

20. Prendergast GC, Ziff EB. Mbh 1: a novel gelsolin/severinrelated protein which binds actin in vitro and exhibits nuclear localization in vivo. EMBO J 1991;10:757-66.

21. Huang S, Chi Y, Qin Y, et al. CAPG enhances breast cancer metastasis by competing with PRMT5 to modulate STC-1 transcription. Theranostics 2018;8:2549-64.

22. Zandberga E, Zayakin P, Abols A, et al. Depletion of carbonic anhydrase IX abrogates hypoxia-induced overexpression of stanniocalcin-1 in triple negative breast cancer cells. Cancer Biol Ther 2017;18:596-605.

23. Chang AC, Doherty J, Huschtscha LI, et al. STC1 expression is associated with tumor growth and metastasis in breast cancer. Clin Exp Metastasis 2015;32:15-27.

24. Bendinelli P, Maroni P, Matteucci E, et al. Epigenetic regulation of $\mathrm{HGF} / \mathrm{Met}$ receptor axis is critical for the outgrowth of bone metastasis from breast carcinoma. Cell Death Dis 2017;8:e2578.

25. Maroni P, Matteucci E, Bendinelli P, et al. Functions and Epigenetic Regulation of Wwox in Bone Metastasis from Breast Carcinoma: Comparison with Primary Tumors. Int J Mol Sci 2017;18.

26. Yeung HY, Lai KP, Chan HY, et al. Hypoxia-inducible factor-1-mediated activation of stanniocalcin-1 in human cancer cells. Endocrinology 2005;146:4951-60.

27. Law AY, Ching LY, Lai KP, et al. Identification and characterization of the hypoxia-responsive element in human stanniocalcin-1 gene. Mol Cell Endocrinol 2010;314:118-27.

28. He LF, Wang TT, Gao QY, et al. Stanniocalcin-1 promotes tumor angiogenesis through up-regulation of VEGF in gastric cancer cells. J Biomed Sci 2011;18:39.

29. Law AY, Wong CK. Stanniocalcin-1 and -2 promote angiogenic sprouting in HUVECs via VEGF/VEGFR2 and angiopoietin signaling pathways. Mol Cell Endocrinol 2013;374:73-81.

30. Olsen HS, Cepeda MA, Zhang QQ, et al. Human stanniocalcin: a possible hormonal regulator of mineral metabolism. Proc Natl Acad Sci U S A 1996;93:1792-6.

31. Murai R, Tanaka M, Takahashi Y, et al. Stanniocalcin-1 promotes metastasis in a human breast cancer cell line through activation of PI3K. Clin Exp Metastasis 2014;31:787-94.

32. Munster PN, Thurn KT, Thomas S, et al. A phase II study of the histone deacetylase inhibitor vorinostat combined with tamoxifen for the treatment of patients with hormone therapy-resistant breast cancer. Br J Cancer 2011;104:1828-35. 
33. Chen S, Ye J, Kijima I, et al. The HDAC inhibitor LBH589 (panobinostat) is an inhibitory modulator of aromatase gene expression. Proc Natl Acad Sci U S A 2010;107:11032-7.

34. Chiu HW, Yeh YL, Wang YC, et al. Combination of the novel histone deacetylase inhibitor YCW1 and radiation induces autophagic cell death through the downregulation of BNIP3 in triple-negative breast cancer cells in vitro and in an orthotopic mouse model. Mol Cancer 2016;15:46.

35. Schech A, Kazi A, Yu S, et al. Histone Deacetylase Inhibitor Entinostat Inhibits Tumor-Initiating Cells in

Cite this article as: Brown JE, Wood SL. Novel mediators of breast cancer bone metastasis-insights from studies of gene-regulation and the global proteome. Ann Transl Med 2018;6(Suppl 1):S71. doi: 10.21037/atm.2018.10.41
Triple-Negative Breast Cancer Cells. Mol Cancer Ther 2015;14:1848-57.

36. Cha YJ, Jung WH, Koo JS. Expression of DNA methylation-related proteins in metastatic breast cancer. Neoplasma 2017;64:412-20.

37. Zhao S, Geybels MS, Leonardson A, et al. EpigenomeWide Tumor DNA Methylation Profiling Identifies Novel Prognostic Biomarkers of Metastatic-Lethal Progression in Men Diagnosed with Clinically Localized Prostate Cancer. Clin Cancer Res 2017;23:311-9. 